\title{
Atuação da Fisioterapia na reabilitação de pacientes pós-COVID-19: uma revisão
}

\section{integrativa}

\author{
The role of Physiotherapy in the rehabilitation of post-COVID-19 patients: an integrative review \\ El papel de la Fisioterapia en la rehabilitación de pacientes post-COVID-19: una revisión
}

integradora

Recebido: 08/10/2021 | Revisado: 13/11/2021 | Aceito: 13/11/2021 | Publicado: 20/11/2021

\author{
Victor Luí Brito Macedo \\ ORCID: https://orcid.org/0000-0003-3617-7273 \\ Centro Universitário da Amazônia, Brasil \\ E-mail: lui.macedo@ hotmail.com \\ Vanessa Andrea Sousa da Silva \\ ORCID: https://orcid.org/0000-0001-9346-0302 \\ Centro Universitário da Amazônia, Brasil \\ E-mail: vanhessa.andrea@gmail.com \\ Maiara Silvana Salgado Batista \\ ORCID: https://orcid.org/0000-0003-2884-8794 \\ Centro Universitário da Amazônia, Brasil. \\ E-mail: maiarasilvana@hotmail.com
}

\begin{abstract}
Resumo
Introdução: A COVID-19 é uma doença infecciosa causada pelo SARS-CoV-2, tornando-se uma emergência global. O coronavírus pode ocasionar sintomas leves ou graves, dependendo do grau de infecção, sendo eles tosse seca, febre, mialgias e dificuldades respiratórias. Sua transmissão acontece de forma rápida, por meio de gotículas respiratórias, que podem ser expelidas através da fala, espirro e tosse, até mesmo por objetos contaminados. A gravidade da doença se relaciona a indivíduos do grupo de risco, que indivíduos acometidos por complicações do COVID-19: tais como fumantes, obesos, portadores de doenças cardiovasculares, comorbidades subjacentes, doença hepática, câncer etc. Além das doenças do sistema respiratório. Neste contexto de pandemia, a abordagem fisioterapêutica se mostrou eficaz e de grande importância, tanto no início do tratamento como na fase de recuperação da infecção causada pela COVID-19. Objetivo: Realizar uma revisão bibliográfica sobre a atuação da Fisioterapia na reabilitação de pacientes pós-COVID-19. Metodologia: estudo de revisão bibliográfica de literatura, as bases de dados escolhidas para a realização da pesquisa foram: Google Acadêmico, PubMed e Science Direct. Os critérios de inclusão foram: pesquisas que abordam sobre a COVID-19, com foco à reabilitação fisioterapêutica em pacientes pós-COVID-19, publicadas entre 2020 e 2021, nos idiomas: português e inglês. Resultados: Após a análise e discussão dos dados, 08 estudos evidenciaram resultados positivos no processo de reabilitação do paciente pós-COVID. Considerações Finais: Concluindo que a Fisioterapia tem um papel principal na melhora da função respiratória e consequentemente na qualidade de vida do paciente.
\end{abstract}

Palavras-chave: COVID-19; Fisioterapia; Reabilitação.

\begin{abstract}
COVID-19 is an infectious disease caused by SARS-CoV-2, making it a global emergency. Coronaviruses can cause mild or severe symptoms, depending on the degree of infection, such as dry cough, fever, myalgia and breathing difficulties. Its transmission happens quickly, through respiratory droplets, which can be expelled through speech, sneezing and coughing, even by contaminated objects. The severity of the disease is related to individuals in the risk group, which are individuals affected by complications of COVID-19: such as smokers, obese people, people with cardiovascular disease, underlying comorbidities, liver disease, cancer, etc. In addition to diseases of the respiratory system. In this pandemic context, the physical therapy approach proved to be effective and of great importance, both in the beginning of treatment and in the recovery phase of the infection caused by COVID-19. Objective: Conduct a literature review on the role of Physiotherapy in the rehabilitation of post-COVID-19 patients. Methodology: study of literature review, the databases chosen for the research were: Academic Google, PubMed and Science Direct. Inclusion criteria were research addressing COVID-19, focusing on physical therapy rehabilitation in post-COVID-19 patients, published between 2020 and 2021, in Portuguese and English. Results: After analyzing and discussing the data, 08 studies showed positive results in the rehabilitation process of the post-COVID patient. Final Considerations: Concluding that Physiotherapy has a main role in improving respiratory function and consequently in the patient's quality of life.
\end{abstract}

Keywords: COVID-19; Physiotherapy; Rehabilitation. 


\begin{abstract}
Resumen
COVID-19 es una enfermedad infecciosa causada por el SARS-CoV-2, lo que la convierte en una emergencia mundial. Los coronavirus pueden causar síntomas leves o graves, según el grado de infección, como tos seca, fiebre, mialgia y dificultad para respirar. Su transmisión se produce de forma rápida, a través de gotitas respiratorias, que pueden ser expulsadas por el habla, estornudos y tos, incluso por objetos contaminados. La gravedad de la enfermedad está relacionada con los individuos del grupo de riesgo, que son individuos afectados por complicaciones del COVID19: como fumadores, personas obesas, personas con enfermedad cardiovascular, comorbilidades subyacentes, enfermedad hepática, cáncer, etc. Además de las enfermedades respiratorias. En este contexto pandémico, el abordaje de la Fisioterapia demostró ser eficaz y de gran importancia, tanto en el inicio del tratamiento como en la fase de recuperación de la infección por COVID-19. Objetivo: Realice una revisión de la literatura sobre el papel de la fisioterapia en la rehabilitación de pacientes post-COVID-19. Metodología: estudio de revisión de la literatura, las bases de datos elegidas para la investigación fueron: Google Académico, PubMed, ScienceDirect. Los criterios de inclusión fueron: investigación sobre COVID-19, centrada en la rehabilitación de Fisioterapia en pacientes postCOVID-19, publicada entre 2020 y 2021, en portugués e inglés. Resultados: Tras analizar y discutir los datos, 08 estudios arrojaron resultados positivos en el proceso de rehabilitación del paciente postsocial. Consideraciones finales: Concluyendo que la Fisioterapia tiene un papel fundamental en la mejora de la función respiratoria y consecuentemente en la calidad de vida del paciente.
\end{abstract}

Palabras clave: COVID-19; Fisioterapia; Rehabilitación.

\title{
1. Introdução
}

A COVID-19 é uma doença infecciosa causada pelo novo coronavírus, denominado SARS-CoV-2, descoberto pela primeira vez no mês de dezembro de 2019, na cidade de Wuhan, na China. Com uma rápida evolução do contágio da doença por diversos outros países a Organização Mundial da Saúde (OMS) oficializou em 11 de março de 2020 como a pandemia do novo coronavírus (Moreno et al, 2021).

Entende-se atualmente que a transmissão do SARS-CoV-2 é dada por inalação ou contato diretamente com gotículas infectadas, entre um período de 1 a 14 dias. Existem casos em que pacientes acometidos pela patologia podem somente transmitir a doença, por serem assintomáticos, e não apresentarem nenhum tipo de sintoma (Estevão, 2020).

Com uma análise profunda e detalhada de todos os primeiros casos, elencou-se os primeiros sintomas comuns da COVID-19: cansaço, febre e tosse seca. Uma parte dos pacientes podem ter dores, congestão nasal, dor de cabeça, conjuntivite, dor de garganta, diarreia, perda de paladar ou olfato, erupção cutânea na pele ou descoloração dos dedos das mãos ou dos pés. As causas para existência para pacientes assintomáticos, citados anteriormente ainda não foram identificados (Ferrari, 2020).

Uma parcela dos pacientes após o período de infecção, podem apresentar sequelas que poderão perdurar por um curto período ou serem permanentes. Essas sequelas não são somente no âmbito respiratório, mas também podem atingir outras funcionalidades do corpo, já que a COVID-19 não ataca somente o sistema respiratório, mas também a funcionalidade de outros sistemas (Ferrari, 2020).

Pessoas com fatores de risco são os principais indivíduos acometidos por complicações do COVID-19: tais como fumantes, obesos, portadores de doenças cardiovasculares, comorbidades subjacentes, doença hepática, câncer etc. Além das doenças do sistema respiratório, algumas das principais complicações relatadas, são neurológicas, tais como alteração no paladar (hipogeusia) e olfato (anosmia), AVC (acidente vascular cerebral), encefalopatia. Muitos dos casos relatam manifestações neurológicas, enquanto não manifestam sintomas respiratórios (OPAS/OMS, 2020).

No fim do mês de fevereiro de 2020, houve o primeiro caso da COVID-19 no Brasil, dados destacam que a vinda do vírus no território nacional pode ter acontecido de diversas formas. Com interesse de conter o avanço e a propagação da doença, protocolos foram desenvolvidos e aprovados pela OMS. Esses protocolos resumem-se em manter os pacientes com sintomas leves isolados em casa, para tentar diminuir o fluxo de contaminados nos hospitais, além de determinar a quarentena (Silva et al, 2021).

Após melhora na recuperação dos efeitos respiratórios agudos devido à doença, a CIF (Classificação Internacional de Funcionalidade, Incapacidade e Saúde), é necessário dar andamento à reabilitação com um auxílio de um fisioterapeuta, 
realizando a avalição conforme o estado do paciente, montando um plano de tratamento individual para uma melhor reabilitação. (Sheehy, 2020).

A reabilitação é a chave principal de recuperação, isso porque se torna essencial para a melhora nas funções cognitivas e físicas a fim de reduzir os riscos na funcionalidade do indivíduo. (Sales, 2020).

O fisioterapeuta tem grande importância neste cenário, desde a atuação nas linhas de frente, na prevenção e reabilitação nos casos graves da infecção, no auxílio para melhor funcionalidade, e limitações nas atividades de vida diária do indivíduo. (Sales, 2020).

Com destaque deste profissional junto com a equipe multidisciplinar dentro do cenário da doença, este estudo objetiva-se a mostrar a importância da Fisioterapia durante a reabilitação dos pacientes pós-COVID-19.

\section{Metodologia}

O presente estudo consiste em uma revisão integrativa da literatura caracterizado por uma abordagem metodológica sistemática, ordenada, abrangente e secundária, com análise de estudos primários. A forma criteriosa para a realização da Revisão Integrativa segue uma busca rigorosa, uma análise e síntese dos dados, chegando à decisão final sobre determinado problema para no fim qualificá-lo (Casarin et al., 2020).

Os estudos utilizados se encontram indexados nas seguintes bases de dados: Google Acadêmico, PubMed e ScienceDirect. Adotaram-se os seguintes critérios de inclusão: artigos publicados no período de 2020 a 2021 em idiomas inglês e português somente; artigos de acesso on-line livre, artigos relacionados com intervenção fisioterapêutica na reabilitação de pacientes pós-COVID-19. Foram excluídos estudos do tipo revisão bibliográfica, dissertações, teses, monografias, diretrizes clínicas, estudos sistemáticos e resumos que não disponibilizavam trabalhos completos e trabalhos clínicos não finalizados.

A busca efetiva dos artigos se deu através dos seguintes descritores do assunto: COVID-19 ou COVID-19 Pandemic, physiotherapy, rehabilitation, respectivamente, COVID-19 ou pandemia do COVID-19, Fisioterapia, reabilitação. Utilizando a conexão entre eles através do operador booleano AND e OR.

\section{Resultados e Discussão}

A busca dos estudos foi realizada nas três bases de dados, ao total 955 foram encontrados. Nas buscas realizadas nas bases de dados ScienceDirect foram encontrados 69 artigos no total, após a leitura dos títulos foram selecionados apenas 12 estudos no total, após a leitura na íntegra apenas 3 entraram nesta revisão.

Nas buscas realizadas nas bases de dados Google Acadêmico foram encontrados 68 estudos no total, após a leitura criteriosa dos títulos foram selecionados 8 no total, após análise dos títulos apenas 2 foram utilizados.

Nas buscas realizadas na base PubMed, foram encontrados 818 resultados no total, por meio da leitura dos títulos apenas 3 foram selecionados, após avaliação dos artigos apenas 2 entraram nesta revisão.

Um total de 8 estudos foram incluídos nos resultados desta pesquisa. 
Tabela 1 - Resultados dos estudos publicados nos anos de 2020 a 2021.

\begin{tabular}{|c|c|c|c|c|}
\hline Objetivo & $\begin{array}{c}\text { Autores } \\
\text { (ano) }\end{array}$ & Amostra & Terapia Empregada & Conclusão \\
\hline $\begin{array}{l}\text { Descrever a experiência } \\
\text { de quatro casos, de } \\
\text { diferentes gravidades, } \\
\text { que realizaram um } \\
\text { programa de reabilitação } \\
\text { cardiopulmonar pós- } \\
\text { COVID-19. }\end{array}$ & $\begin{array}{c}\text { Tozato et } \\
\text { al., (2021). }\end{array}$ & $\begin{array}{l}4 \text { pacientes, } \\
\text { sendo } 2 \\
\text { mulheres e } 2 \\
\text { homens, com } \\
\text { faixa de } 43 \text { a } \\
72 \text { anos. }\end{array}$ & $\begin{array}{c}\text { Foi realizado um protocolo de } \\
\text { reabilitação Cardiopulmonar por } 3 \\
\text { meses pós-COVID. Realizadas: } \\
\text { exercício aeróbicos, exercícios } \\
\text { resistidos, Teste de caminhada de } 6 \\
\text { minutos, teste de força de preensão } \\
\text { manual e teste de 1RM para cada grupo } \\
\text { muscular. }\end{array}$ & $\begin{array}{l}\text { O programa de exercícios físicos } \\
\text { baseado em princípios da } \\
\text { reabilitação cardiovascular e } \\
\text { pulmonar apresentou impacto } \\
\text { positivo nos casos acompanhados, } \\
\text { com melhora da capacidade } \\
\text { funcional, mesmo com a } \\
\text { variabilidade da gravidade dos } \\
\text { casos pós-COVID-19. }\end{array}$ \\
\hline $\begin{array}{l}\text { Avaliar a capacidade } \\
\text { funcional de exercício } \\
\text { nos pacientes com } \\
\text { COVID } 19 \text { grave e } \\
\text { avaliar o efeito de um } \\
\text { programa de } \\
\text { telereabilitação no pós- } \\
\text { COVID. }\end{array}$ & $\begin{array}{c}\text { Martin et } \\
\text { al., (2021). }\end{array}$ & $\begin{array}{l}103 \text { pacientes } \\
\text { internados } \\
\text { para COVID- } \\
19 \text { grave ou } \\
\text { crítico. }\end{array}$ & $\begin{array}{l}\text { Estudo realizado por duas fases: (1) } \\
\text { avaliação da capacidade de exercício } \\
\text { funcional durante } 2 \text { meses; }(2) \\
\text { avaliação sobre os efeitos da } \\
\text { telereabilitação nos pacientes. }\end{array}$ & $\begin{array}{l}\text { Pacientes graves ou críticos } \\
\text { internados para COVID-19 têm } \\
\text { baixa capacidade funcional de } \\
\text { exercício na alta e a recuperação } \\
\text { após } 3 \text { meses. Mais importante, } \\
\text { verificou-se a viabilidade e a } \\
\text { segurança de um simples programa } \\
\text { de telereabilitação nesses pacientes } \\
\text { quando imediatamente iniciado na } \\
\text { alta. Este programa foi capaz de } \\
\text { melhorar substancialmente a } \\
\text { recuperação funcional após três } \\
\text { meses. }\end{array}$ \\
\hline $\begin{array}{c}\text { Mostrar a reabilitação } \\
\text { pulmonar em pacientes } \\
\text { que sofreram de COVID- } \\
19 .\end{array}$ & $\begin{array}{l}\text { Spielmanns } \\
\text { et al., } \\
\text { (2021). }\end{array}$ & $\begin{array}{l}99 \text { pacientes } \\
\text { pós-COVID } \\
\text { foram } \\
\text { admitidos na } \\
\text { reabilitação } \\
\text { pulmonar. }\end{array}$ & $\begin{array}{l}\text { Exercícios de força 3-4 vezes por } \\
\text { semana, individualmente. A escala de } \\
\text { Borg modificada usada para definir a } \\
\text { intensidade do exercício. Exercícios } \\
\text { para grandes grupos musculares. } \\
\text { Treinamento e relaxamento muscular } \\
\text { inspiratório (relaxamento muscular } \\
\text { progressivo). Fisioterapia respiratória, } \\
\text { controle da respiração (respiração com } \\
\text { lábios franzidos, mobilização de } \\
\text { secreção e respiração diafragmática), } \\
\text { técnicas de economia de energia e } \\
\text { exercícios de tosse controlada. } \\
\end{array}$ & $\begin{array}{l}\text { O estudo fornece evidências de que } \\
\text { a reabilitação pulmonar abrangente } \\
\text { pós-aguda está associada a } \\
\text { melhorias clínicas e funcionais } \\
\text { significativas em indivíduos que } \\
\text { sofreram de COVID-19 grave e } \\
\text { destaca a importância da } \\
\text { reabilitação pós-aguda para a } \\
\text { recuperação de COVID-19. }\end{array}$ \\
\hline $\begin{array}{c}\text { Mostrar a intervenção } \\
\text { fisioterapêutica em } \\
\text { indivíduos com COVID- } \\
19 .\end{array}$ & $\begin{array}{l}\text { Lee et al., } \\
(2020) \text {. }\end{array}$ & $\begin{array}{l}9 \text { pacientes, } \\
\text { com média de } \\
\text { idade de } 66 \\
\text { anos, sendo } 6 \\
\text { do sexo } \\
\text { masculino e } 3 \\
\text { do feminino. }\end{array}$ & $\begin{array}{l}\text { Pequenas sessões com vários intervalos } \\
\text { de descanso entre as séries de } \\
\text { exercícios. Uma abordagem gradual } \\
\text { também foi usada durante a } \\
\text { mobilização, oxigênio suplementar } \\
\text { adicional foi fornecido, se necessário. } \\
\text { Os sinais vitais foram monitorados } \\
\text { continuamente em todas as sessões para } \\
\text { garantir a detecção de alterações na } \\
\text { oxigenação. }\end{array}$ & $\begin{array}{l}\text { A conclusão é baseada em um } \\
\text { único centro em Cingapura. } \\
\text { Considerando que o NCID é o } \\
\text { maior centro de Cingapura. Nosso } \\
\text { achado é provavelmente } \\
\text { representativo dos indivíduos com } \\
\text { infecção por COVID-19. No } \\
\text { entanto, a necessidade de } \\
\text { intervenção fisioterapêutica } \\
\text { respiratória pode ser diferente } \\
\text { devido às diferenças regionais no } \\
\text { perfil dos pacientes, prevalência de } \\
\text { comorbidades e práticas } \\
\text { fisioterapêuticas. }\end{array}$ \\
\hline $\begin{array}{l}\text { Investigar os efeitos do } \\
\text { treinamento de } \\
\text { reabilitação respiratória } \\
\text { de } 6 \text { semanas na função } \\
\text { respiratória, QV, } \\
\text { mobilidade e função } \\
\text { psicológica em pacientes } \\
\text { idosos com COVID-19. }\end{array}$ & $\begin{array}{l}\text { Liu et al., } \\
(2020) \text {. }\end{array}$ & $\begin{array}{l}72 \text { pacientes, } \\
\text { idade } \geq 65 \\
\text { anos, onde } 36 \\
\text { pacientes } \\
\text { foram } \\
\text { submetidos a } \\
\text { fazer à } \\
\text { reabilitação } \\
\text { respiratória e } \\
\text { o restante a } \\
\text { outros tipos de } \\
\text { reabilitação } \\
\text { durante } 6 \\
\text { semanas. }\end{array}$ & $\begin{array}{l}\text { Foram medidos os seguintes desfechos: } \\
\text { testes de função pulmonar, incluindo } \\
\text { plethysmografia e difusão da } \\
\text { capacidade pulmonar para monóxido de } \\
\text { carbono (DLCO), testes funcionais } \\
\text { (teste de distância de } 6 \text { min), avaliações } \\
\text { de qualidade de vida (QV) (escores de } \\
\text { SF-36), atividades de vida diária } \\
\text { (Medida de Independência Funcional, } \\
\text { escores FIM) e testes de estado mental } \\
\text { (escores de ansiedade SAS e SDS). }\end{array}$ & $\begin{array}{c}\text { A reabilitação respiratória de seis } \\
\text { semanas pode melhorar a função } \\
\text { respiratória, a QV e a ansiedade de } \\
\text { pacientes idosos com COVID-19, } \\
\text { mas tem pouca melhora } \\
\text { significativa na depressão em } \\
\text { idosos. }\end{array}$ \\
\hline
\end{tabular}




\begin{tabular}{|c|c|c|c|c|}
\hline $\begin{array}{c}\text { Mostrar a versão } \\
\text { modificada de exercícios } \\
\text { de reabilitação que visam } \\
\text { melhorar a função } \\
\text { pulmonar. }\end{array}$ & $\begin{array}{l}\text { Zha et al., } \\
(2020) .\end{array}$ & $\begin{array}{l}60 \text { pacientes, } \\
\text { com idade } \\
\text { mediana de } 54 \\
\text { anos. }\end{array}$ & $\begin{array}{l}\text { Exercício de reabilitação modificado } \\
\text { (MRE). } 8 \text { seções da arte marcial } \\
\text { chinesa. Alongamento de tórax e } \\
\text { ombro. Elevação do calcanhar em pé e } \\
\text { acupressão da parte superior do corpo } 2 \\
\text { séries de } 12 \text { reptições. Rotação da parte } \\
\text { superior do corpo } 1 \text { série de } 4 \\
\text { repetições e massagem com acupressão } \\
\text { nas mãos } 3 \text { séries de } 12 \text { repetições. }\end{array}$ & $\begin{array}{c}\text { Foram usados exercícios de } \\
\text { reabilitação modificados para o } \\
\text { trastamento de pacientes com } \\
\text { COVID-19. A taxa de remissão } \\
\text { mostrou melhora nos sintomas } \\
\text { respiratórios. Utilizou-se o MRE } \\
\text { para suavizar a respiração e } \\
\text { melhorar o processo de } \\
\text { expectoração nos casos de COVID- } \\
19 \text { leves. }\end{array}$ \\
\hline $\begin{array}{l}\text { Verificar a eficácia do } \\
\text { treinamento respiratóio e } \\
\text { reabilitação remota. }\end{array}$ & Li (2020). & $\begin{array}{l}4 \text { pacientes } \\
\text { foram } \\
\text { submetidos a } \\
\text { reabilitação } \\
\text { motora. }\end{array}$ & $\begin{array}{c}\text { Treinamento respiratório e treinamento } \\
\text { aeróbio. Exercícios tradicionais (Tai-ji, } \\
\text { Ba-duan-jin). Treinamento de } \\
\text { deglutição, apoio nutricional e ajuda } \\
\text { psicológica. }\end{array}$ & $\begin{array}{l}\text { Após a COVID-19 houve a } \\
\text { aplicação da medicina de } \\
\text { reabilitação remota. Abordagens de } \\
\text { reabilitação foram aceleradas, o } \\
\text { que incluio dispositivos vestíveis, } \\
\text { APPs de telefones celulares. }\end{array}$ \\
\hline $\begin{array}{l}\text { Reabilitação de pacientes } \\
\text { com COVID-19. }\end{array}$ & $\begin{array}{l}\text { Sandro et } \\
\text { al., }(2020) \text {. }\end{array}$ & $\begin{array}{l}300-400 \\
\text { pacientes } \\
\text { internados no } \\
\text { Hospital San } \\
\text { Raffaele. Com } \\
\text { média de } 70 \\
\text { anos, durante } \\
\text { o período de } \\
\text { março a abril } \\
\text { de } 2020 .\end{array}$ & $\begin{array}{l}\text { Fisioterapia respiratória; manejo da } \\
\text { ventilação não-invasiva; manejo da } \\
\text { postura/terapia posicional; mobilização } \\
\text { passiva e ativa do paciente; avaliação } \\
\text { de critérios respiratórios e motores. } \\
\text { Recuperação da mobilidade a } \\
\text { autonomia. Telereabilitação. }\end{array}$ & $\begin{array}{l}\text { De acordo com a experiência de } \\
\text { San Raffaele. Cerca de } 80 \% \text { dos } \\
\text { pacientes apresentaram escores } \\
\text { anormais no Mini-Exame Do } \\
\text { Estado Mental ou Avaliação } \\
\text { Cognitiva de Montreal na admissão } \\
\text { na unidade de reabilitação COVID- } \\
19 \text { e a maioria dos pacientes } \\
\text { recuperou valores normais na alta } \\
\text { hospitalar. }\end{array}$ \\
\hline
\end{tabular}

Legenda: FR: Fisioterapia Respiratória. NCID: Centro de Doenças Infecciosas. Fonte: Macedo, Silva, Batista. (2021).

A COVID-19 pode levar a uma série de apresentações clínicas podendo acometer, além dos pulmões, os demais sistemas, sendo eles: cardiovascular, neurológico, hematológico e urinário. Na maioria, a infecção pela COVID-19 pode levar à febre $(88,7 \%)$, tosse $(57,6 \%)$ e dispneia $(45,6 \%)$ sendo os sintomas mais comumente relatados em revisão sistemática recente e meta-análise (Simpson \& Robinson, 2020).

Em pessoas com mais de 65 anos que apresentem comorbidades, a infecção pode gerar consequências graves. Estimase que 20,3\% pacientes necessitem de internação em unidades de terapia intensiva (UTI), sendo um dos motivos à Síndrome de desconforto respiratório aguda $(32,8 \%)$. Os pacientes podem não devolver lesão hepática aguda, lesão renal, lesão cardíaca aguda e choque séptico. Foi relatado o índice de óbitos por pacientes hospitalizados, sendo (71\%) por síndrome respiratória aguda e coagulopatia intravascular disseminada, se tornando a principal causa de mortes após a infecção do COVID-19. (Simpson \& Robinson, 2020).

Após a internação hospitalar grande parte dos indivíduos poderão apresentar sequelas após a recuperação. As sequelas respiratórias causas pela COVID-19 afeta principalmente os pulmões. Após alta hospitalar, no período de 6 à 8 semanas, mostrasse fraqueza muscular de $6 \%$ a $20 \%$. Dependendo do grau de infecção, destacam-se sequelas variadas, como volume e capacidade pulmonar reduzida, alterações nos exames de imagem, limitação durante a execução de exercícios e redução na capacidade funcional. (Avila, et al, 2020).

O estudo de Tozato et al (2021), mostrou quatro casos com diferentes classificações de gravidade e resultados após a infecção por COVID-19. O plano de reabilitação é direcionado segundo os princípios da reabilitação pulmonar e cardiovascular, enfatizando nas possíveis sequelas pulmonares, como diminuição da saturação e dispneia. A diminuição da saturação foi verificada em dois casos, onde houve queixas de dispneia durante o treinamento. De forma progressiva. Todos os quatro casos apresentaram efeitos positivos, se beneficiando dos treinos aeróbicos e resistidos, ambos avaliados pelo teste de repetição máxima. Foi constatado redução dos sintomas, melhora durante a avaliação de esforço submáximo, mesmo com variabilidades em casos graves após a COVID-19. 
Em seu estudo Martin et al (2021), afirma que pacientes que foram internados devido a COVID-19 possuem baixa capacidade funcional durante a realização de exercícios na alta, afirmou também que a recuperação após três meses ainda não mostra excelentes resultados. Por outro lado, verificou a eficácia de um simples programa de telereabilitação, sendo este supervisionado por um fisioterapeuta experiente no ramo. Esta avaliação foi feita por 2 fases, onde a primeira buscava avaliar a capacidade do exercício funcional, em segundo utilizar a telereabilitação. Segundo Salas et al (2021), a Telerreabilitação mostrou resultados positivos na fadiga e dispneia, e foi eficaz na melhora da capacidade física e qualidade de vida.

O teste de 1 min sentado (STST) foi utilizado na primeira fase. Para a segunda fase, foi empregado o uso de videoconferências para a supervisão do paciente, as sessões aconteciam em seus respectivos domicílios, 2 vezes na semana, por seis semanas, com 50 minutos de duração. Exercícios de resistência, fortalecimento eram aplicados por $30 \mathrm{~min}$, a intensidade dos exercícios era avaliada com auxílio da escala de Borg. O treinamento da musculatura do corpo era realizado com material domiciliar, com um total 2-3 séries de 8-12 repetições para cada exercício.

Segundo Fuglebjerg, (2020) os fisioterapeutas utilizaram como método o Teste de Caminhada de 6 minutos (TC6M) para analisar possíveis hipoxemia silenciosa nos pacientes, identificando a presença de trombose, com a finalidade de descartar os riscos para o paciente, e em seguida dar a alta hospitalar.

No estudo de Lee et al (2020), a taxa de pacientes levados para Fisioterapia é baixa. Apesar de que seja provável que alguns dos pacientes que podem se favorecer de intervenções fisioterapêuticas que não foram direcionados ao serviço, esse achado também pode ser concedido a vários outros fatores. Todos os enfermos com infecção por COVID-19 foram internados em hospitais em Cingapura como parte do esforço de contenção do governo. A maior parte desses pacientes eram assintomáticos ou tinham sintomas leves e, portanto, não requer tratamento fisioterapêutico.

Além disso, a mobilização precoce realizada por enfermeiras (um padrão de atendimento em instituição) foi executada a pacientes internados em UTI. Por fim, desde o início do surto, gravações de exercícios foram cedidos a todos os pacientes nas enfermarias médicas gerais para certificar que eles continuassem ativos durante seu isolamento. Com isso, este estudo pode não ser generalizável para outros centros e deve ser interpretado com cautela. Porém, os achados deste relato de caso contribuem para uma nova visão sobre a apresentação clínica de pacientes com COVID-19. Os fisioterapeutas devem levar em consideração essas recomendaç̃os ao fornecer reabilitação para esse grupo de pacientes. (Lee et al. 2020).

No estudo randomizado controlado de Liu et al (2020), feito com 72 pacientes idosos de idade $\geq 65$ anos, com diagnóstico exclusivo de COVID-19, e sem qualquer outra doença respiratória ou cardíaca anterior; dos quais, apenas 36 pacientes foram submetidos ao programa de reabilitação respiratória. Este estudo conteve as seguintes intervenções durante 6 semanas: treino dos músculos respiratórios utilizando (Threshold PEP; Philips Co.) com 3 séries e 10 repetições a cada série e parâmetros de $60 \%$ da pressão expiratória máxima e descanso de 1 minuto entre as séries, treino de tosse (três séries de 10 tosses ativas), treinamento diafragmático (30 contrações diafragmáticas voluntárias máxima em posição supina, com peso de 1 á $3 \mathrm{~kg}$ em parede abdominal anterior para resistência a descida diafragmática), e exercícios de alongamento da musculatura respiratória. Ao final do estudo foi constatado melhora significativa na capacidade de exercícios destes pacientes, além da diminuição positiva do quadro de ansiedade e depressão.

No estudo de Zha et al (2020), eles verificaram que foram notificados 60 casos confirmados de COVID-19 leves para análise. 39 do sexo masculino, com idade de 54 anos, e intervalo de 38-62. Houve uma alta gravidade no desenvolvimento dos sintomas respiratórios. A dificuldade para tossir e dispneia tiveram como porcentagem 41,7\%, diminuindo conforme o tempo. Apenas $15 \%$ dos pacientes apresentaram como sintoma restante a dispneia. Diversas terapias de reabilitação foram feitas promovendo facilidade ao processo de recuperação de pacientes com doenças respiratórias.

Segundo o estudo Li (2020), os tratamentos para pacientes leves são realizados por exercícios envolvendo o treinamento respiratório e aeróbio leve. Deve-se prestar atenção nas medidas tomadas de reabilitação na fase aguda devido as 
secreções em casos graves, tendo em vista que o principal dano da COVID-19 é o excesso de expectoração alveolar, o problema da troca de ar e o risco inflamatório. Uma pesquisa feita no Hospital de Integrated Chinese and Western Medicine, notou que 63,6\% dos pacientes leves que iriam receber alta apresentavam dificuldade para dormir, resistência baixa ao praticar exercício, falta de ar leve e ansiedade. Devido a quarente adotaram a reabilitação remota que foi muito útil para promover um tratamento doméstico e comunitário.

De acordo com Sandro et al (2020), o manejo postural, a mobilização passiva e ativa, associados à reabilitação cognitiva e respiratória, reduziu o tempo de internação dos pacientes e mostrou sinais positivos de melhora nas funções cognitivas, especificamente nos idosos, que tinham apresentados scores anormais durante a Avaliação cognitiva de Montreal ou do Mini-exame de estado mental.

\section{Considerações Finais}

A infecção pela COVID-19 pode acontecer de diversas formas, a gravidade do problema muda conforme cada indivíduo e seu grupo de risco. Após análise dos estudos de forma criteriosa pode-se notar que os diversos meios de reabilitação feitas pela Fisioterapia são eficazes, principalmente após a alta do paciente acometido pela infecção. As técnicas realizadas no pós-COVID mostram sinal positivo no ganho de força, na função motora, na realização de atividades de vida diária, e principalmente na melhora da função respiratória.

A Fisioterapia nos tempos de pandemia tem total necessidade no combate ao COVID-19, apesar de enormes desafios, a atuação teve papel fundamental na promoção da saúde, e ao retorno funcional e motor dos indivíduos curados.

Até o momento, é notória a necessidade de mais pesquisas sobre a COVID-19, evidenciando um corpo sólido sobre as características e principalmente os efeitos de intervenção Fisioterapêutico no pós-COVID para os determinados pacientes, baseando-se nas sequelas e disfunções atuais, como motoras, respiratórias e neurológicas, pois futuramente será necessário o surgimento de novas evidências científicas.

\section{Agradecimentos}

Agradecemos a nossa orientadora, professora e fisioterapeuta Maiara Silvana Salgado Batista pelo apoio durante todas as etapas para a execução deste projeto de pesquisa.

\section{Referências}

Avila, P., et al. (2020). Guia de orientações fisioterapêuticas na assistência ao paciente pós-COVID-19. Belém: UFPA, FFTO, Curso de Fisioterapia, 22.p.

Battaglini, D., et al. (2021). An Experimental Pre-Post Study on the Efficacy of Respiratory Physiotherapy in Severe Critically III COVID-19 Patients. J. Clin. Med. 10, 2139.

Casarin, S., et al. (2020). Tipos de revisão de literatura: considerações das editoras do Journal of Nursing and Health / Types of literature review: considerations of the editors of the Journal of Nursing and Health. (10) i5.19924. Journal of Nursing and Health. 10. 10.15210/jonah.

Estevão, A. (2020). Covid-19. ACTA radiológica portuguesa. Centro Hospitalar e Universitário de Coimbra, 32(1), 5-6.

Ferrari, F. (2020). COVID-19: Dados Atualizados e sua Relação Com o Sistema Cardiovascular. Arq. Bras. Cardiol. 114 , nº5.

Fuglebjerg, N. J. U., et al. (2020). Silent hypoxia in patients with SARS CoV-2 infection before hospital discharge. International Journal of Infectious Diseases, Vol. 99, p. 100-101.

Lee, A. J. Y., et al. (2020). Clinical course and physiotherapy intervention in 9 patients with COVID-19. Physiotherapy. 109, 1-3.

Li, J. (2020). Rehabilitation management of patients with COVID-19: lessons learned from the first experience in China. Eur J Phys Rehabil Med. 56:335-8.

Liu, K., et al. (2020). Respiratory rehabilitation in elderly patients with COVID-19: A randomized controlled study. Complementary therapies in clinical practice. $39,101166$. 
Research, Society and Development, v. 10, n. 15, e93101523115, 2021

(CC BY 4.0) | ISSN 2525-3409 | DOI: http://dx.doi.org/10.33448/rsd-v10i15.23115

Martin, I., et al. (2021). Follow-up of functional exercise capacity in patients with COVID-19: It is improved by telerehabilitation. Original Research. 183, 106438, 01 .

Moreno, J. E., et al. (2021). Fisioterapia respiratoria en la funcionalidad del paciente con COVID-19. Arch Med (Manizales). 21(1):266-278.

OPAS/OMS. (2020). Alerta Epidemiológico Complicações e sequelas da COVID-19. PAHO/WHO.

Salas, M. et al (2021). Effectiveness of a Primary Care Telerehabilitation Program for Post-COVID-19 Patients: A Feasibility Study. J. Clin. Med. 10(19), 4428 .

Sales, E. M. P., et al. (2020). Fisioterapia, Funcionalidade e COVID-19: Revisão integrativa. Cadernos ESP-Revista Científica da Escola de Saúde Pública do Ceará. 14(1), 68-73.

Sandro, I., et al. (2020). Role of rehabilitation department for adult individuals with COVID-19: the experience of the san raffaele hospital of milan Arch. Phys. Med. Rehabil. 101 (9), 1656-1661.

Sheehy, L. M., (2020). Considerations for Postacute Rehabilitation for Survivors of COVID-19. JMIR Public Health Surveill. 6 (2): e19462.

Silva, L. C. O., et al. (2021). Sequelas e reabilitação pós-covid19: revisão de literatura. Revista das Ciências da Saúde e Ciências aplicadas do Oeste BaianoHigia. 6(1):169-184.

Simpson, R., \& Robinson, L. (2020). Rehabilitation after critical illness in people with COVID-19 infection. American journal of physical medicine \& rehabilitation, 18 99(6), 470.

Spielmanns, M., et al (2021). Effects of a Comprehensive Pulmonary Rehabilitation in Severe Post-COVID-19 Patients. International Journal of Environment Research and Public Health. 18, 2695.

Tozato, C., et al. (2021). Reabilitação cardiopulmonar em pacientes pós-COVID-19: série de casos. Revista Brasileira de Terapia Intensiva, 33(1), 167-171.

Zha, L. et al. (2020). Modified rehabilitation exercises for mild cases of COVID-19. Ann Palliat Med. 9(5);3100-3106. 